\title{
Brain-derived neurotrophic factor enhances the therapeutic effect of oleuropein in the lipopolysaccharide-induced models of depression
}

\author{
A-na Xu, Feng Nie \\ Department of Psychiatry, Third Hospital of Heilongjiang Province, China
}

Folia Neuropathol 2021; 59 (3): 249-262

DOI: https://doi.org/10.5114/fn.2021.108550

\begin{abstract}
Major depressive disorder (MDD) is a heterogeneous disease, involving multiple mechanisms and factors, which commonly result in injury to the psychosocial function of the central nervous system, and even suicidality of patients. However, effective treatment for MDD is still lacking. Oleuropein is a newly discovered natural compound extracted from olive leaves, which has a strong antioxidative effect by reducing the production of reactive oxygen species (ROS). Oleuropein also reduces blood pressure in humans and experimental animals, and protects blood vessels. Brain-derived neurotrophic factor (BDNF) is a member of the neurotrophin family, which supports the function of the central nervous system. BDNF plays an important role in the development of the nervous system via the regulation of cellular differentiation, survival neurogenesis and synaptic plasticity; therefore, we hypothesized that overexpression of BDNF might contribute to the therapeutic effect of oleuropein. Here, we first demonstrated that oleuropein reverses depressive-like behaviour and restores the inflammatory response in a mouse lipopolysaccharide (LPS) model of MDD. We further established a cell model of BDNF overexpression and inhibition in SH-SY5Y cells, and found that the concentration of intercellular calcium was increased after treatment with oleuropein combined with BDNF overexpression, which may be mediated by the BDNF-TrkB-CaMKII signalling pathway. In addition, we observed that the expression of neurotrophic factors, including epidermal growth factor (EGF), neurotrophin-3 (NT-3), and neurotrophin-4 (NT-4), was increased, which may be mediated by inhibition of the RhoA-ROCK signalling pathway.
\end{abstract}

Key words: BDNF, oleuropein, $L P S, M D D$.

\section{Introduction}

Major depressive disorder (MDD) is one of the most common diseases leading to disability and loss of quality of life worldwide [13]. A previous study found a relationship between proinflammatory cytokines and MDD, and an increase in the production of interleukin 6 (IL-6), tumour necrosis factor $\alpha$ (TNF- $\alpha$ ), interleukin $1 \beta$
(IL-1 $\beta)$, and C-reactive protein (CRP) was observed in patients with MDD [17,24]. A previous study showed that this effect occurs in a dose-dependent manner, and that higher expression of these factors predicts the subsequent development of depressive symptoms [42]. Oleuropein is a natural product extracted from olive leaves and has drawn much attention from researchers for its biological effects, including antipro- 
liferative, antidiabetic and anti-inflammatory activities [7]. Oleuropein has many biological functions, including the ability to reduce blood pressure through up-regulation of blood nitric oxide synthase inhibitors (L-NAME) [29], and the ability to decrease apoptosis in a mouse model of splanchnic arterial occlusion (SAO) shock [30]. Moreover, oleuropein could decrease the production of interleukin 1 (IL-1) by $80 \%$ in cultured cells [31], and therefore, may be useful in the treatment of MDD. The neurotrophic hypothesis of depression demonstrated that depression is initially caused by a reduction in brain-derived neurotrophic factor (BDNF) and a decrease in neurogenesis, leading to the impairment of synaptic transmission [25]. BDNF belongs to the neurotrophin family, which contains nerve growth factor (NGF), neurotrophin-3 (NT-3), and neurotrophin-4 (NT-4). And a previous study found that BDNF regulates the development, maintenance, and plasticity of the nervous system, and also regulates the apoptosis of neuronal cells [23]. Moreover, BDNF regulates central nervous system (CNS) development in the immature CNS and regulates synaptic homeostasis in the mature CNS [10]. It is well known that mood disorders are closely related to pathophysiological changes in inflammation, particularly depression. In addition, inflammatory-related factors, including CRP, IL-6, IL-1, and TNF- $\alpha$, are elevated in depression models and are regarded as markers of depression [22]. Thus, we speculated that BDNF overexpression might contribute to the therapeutic effect of oleuropein and performed the following experiments to test this hypothesis. Here, using behavioural tests, we found that oleuropein treatment could help to improve the brain function of MDD mice, as well as to reduce the expression and concentration of proinflammatory factors using in vivo experiments. Moreover, the combination of oleuro- pein and BDNF overexpression increased the viability of cells by inhibiting the production of pro-inflammation cytokines using in vitro experiments, and further experiments found that this effect might be mediated by the RhoA/ROCK signalling pathway using in vitro experiments.

\section{Material and methods \\ Animal model of depression}

Forty C57/BL mice aged 6-8 weeks were purchased from the Medical Laboratory Animal Centre. Mice were housed at $22-24^{\circ} \mathrm{C}$ with $50 \%$ humidity, a $12 / 12-\mathrm{h}$ light-dark cycle, and freely available water and food. Mice were divided into four groups as follows: the control group (CG), the lipopolysaccharide (LPS) model group (LG), the oleuropein treatment group (OG) and the LPS model combined with the oleuropein treatment group (LO). In the LPS model group, mice were treated with $5 \mathrm{mg} / \mathrm{kg}$ LPS (No. trrl-smlps, InvivoGen) $24 \mathrm{~h}$ before performing the following experiments through intraperitoneal injection according to a previous study [8], and in oleuropein treatment, mice were treated with $10 \mathrm{mg} / \mathrm{kg}$ oleuropein (No. 12247, Sigma) 60 min before performing the following experiments through intraperitoneal injection according to a previous study [36] (Fig. 1).

\section{Behaviour tests}

\section{Sucrose preference test}

The sucrose preference test (SPT) was performed to evaluate the anhedonic state of mice [46]. Lowgrade stress is a factor contributing to the aetiology of depression. According to this study, stress leads to increased production of corticosteroids, resulting in an

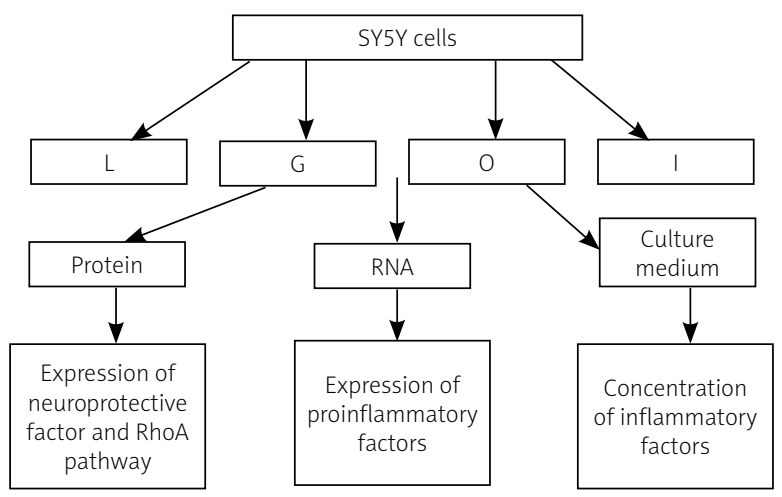

Fig. 1. Scheme of biochemical experiments in this manuscript 
increase in blood sugar levels. High blood sugar in animals leads to a low intake of sucrose, which explains the reduced sucrose preference in mice with MDD. The mice were grouped and treated as described in the manuscript, then, mice were given two bottles of $1 \%$ sucrose on the first day. On the following day, one of the bottles was changed to pure water, and, the mice were fasted for the next $24 \mathrm{~h}$. Subsequently, the mice were given two pre-weighed bottles of drinks, one was a $1 \%$ sucrose solution and the other was drinking water. The mice were given freely available water and food for $1 \mathrm{~h}$, following which, the two bottles were weighed. Sucrose preference (\%) was calculated as follows: sucrose consumption/(sucrose consumption + water consumption) $\times 100 \%$.

\section{Forced swimming test}

The forced swimming test (FST) was performed to evaluate the giving-up-like behaviour of mice [1]. Briefly, mice were forced to swim for 10 min adaptively 1 day before the test. The mice were then placed on a transparent cylinder, and the immobility time in 5 min was recorded using a Super Maze Behaviour Analysis System (Shanghai Xinruan Information Technology Co., Ltd., Shanghai, China).

\section{Open field test}

The open field test (OFT) was performed to measure spontaneous locomotor activity and exploratory behaviour in a novel environment [2]. The experiment was performed in a round open-field apparatus, with a $60 \mathrm{~W}$ bulb positioned above the centre of the apparatus [39]. The mice were placed in the centre of the apparatus to observe their behaviour over a 3-min period, and the number of crossed squares and rearing time were recorded using a Super Maze Behaviour Analysis System (Shanghai Xinruan Information Technology Co., Ltd., Shanghai, China).

\section{Vector construction}

The BDNF knockdown vector was constructed according to a previous study [38]. Briefly, oligos were acquired using the following primers: forward: $5^{\prime}-C A C$ CGTCTACCCACACGCTTCTGTA-3', reverse: 5'-AACTACAGAAGCGTGTGGGTAGAC-3'. The primers were incubated at $95^{\circ} \mathrm{C}$ for $4 \mathrm{~min}$ and $70^{\circ} \mathrm{C}$ for $10 \mathrm{~min}$. Oligos and the CRISPR vector were digested with $\mathrm{BsmBI}$ (No. R0580S, NEB). The BDNF knockdown vector was constructed after annealing with T4 PNK enzyme
(No. M0201S, NEB). The lentivirus vector was constructed after transfection into 293T cells using Lipofectamine 2000 (No. 11668030, Thermo) transfection reagent, and stable expressing cells were screened using $2 \mu \mathrm{g} / \mathrm{ml}$ puromycin (No. P8230, Solarbio). The CDNA of BDNF was obtained using PCR with the following primers: forward: 5'-TCTGACGACGGACATCGGC-3'; reverse: 5'-CCAGCAGAAAGAGTAGAGGAGGC-3'. Primers and the pCDNA3.1 vector were digested with Xbal (No. R0145S, NEB) and Xhol (No. R0146S, NEB), annealed with T4 enzyme, and transfected into SH-SY5Y cells using Lipofectamine 2000 transfection reagent. Stably expressed cells were screened using 800 mg/ml G418 (No. G8161, Solarbio).

\section{Cell model of depression}

SH-SY5Y cells (No. CRL-2266) were purchased from ATCC. The cells were cultured in H-DMEM supplemented with $10 \%$ foetal bovine serum (FBS) (No. $10100, \mathrm{Gibco}$ ) at $37^{\circ} \mathrm{C}$ with $5 \% \mathrm{CO}_{2}$. The cells were treated with $1 \mu \mathrm{g} / \mathrm{ml}$ LPS for $4 \mathrm{~h}$ to construct a major depression model in SH-SY5Y cells [8]. Cells were divided into four groups as follows: the LPS treatment group $(\mathrm{L})$, the LPS treatment combined with oleuropein treatment group $(G)$, the LPS and oleuropein treatment combined with BDNF overexpression group (O) and the oleuropein treatment combined with BDNF inhibition group (I). In the oleuropein treatment group, cells were treated with $10 \mu \mathrm{M}$ oleuropein for $1 \mathrm{~h}$ [26] before performing the following experiments.

\section{MTT assay}

Cells were first seeded into a 96-well plate at a concentration of $1 \times 10^{4}$ cells per well, and then the cells were cultured and grouped as previously described. After treatment, the cells were incubated with MTT for $4 \mathrm{~h}$, and the OD value was detected using SpectraMax iD3 at $570 \mathrm{~nm}$. Viability rate $=\left(O D_{\text {Experiment }}-O D_{\text {Blank }}\right) /\left(O D_{\text {Control }}-O D_{\text {Blank }}\right)$. Each experiment was repeated three times independently.

\section{RNA extraction}

RNA extraction was performed using a Total RNA Extraction Kit. Briefly, cells were cultured and grouped as previously described, lysed with lysis buffer and incubated with chloroform. After centrifugation at 12,000 rpm for $10 \mathrm{~min}$, the water phase of the sample was transferred to an absorption tube. 
The samples were then washed with washing buffer and eluted with elution buffer. The RNA concentration was measured using a Nanodrop 2000. Samples were stored at $-80^{\circ} \mathrm{C}$ until further use.

\section{Quantitative real-time polymerase chain reaction}

The UltraSYBR One-step RT-PCR kit (No. CW0659, CWBio) was used to perform qPCR with 500 pg RNA sample on a StepOnePlus ${ }^{\mathrm{TM}}$ Real-Time PCR System (No. 4376600, Applied Biosystems, Thermo). Briefly, the reaction mixture was prepared as recommended to a total volume of $25 \mu \mathrm{l}$, and the reaction was performed with the following primers: IL-1 $\beta$ : forward: 5'-AGGTGGTGTCGGTCATCGT-3', reverse: 5'-GCTCTCTGTCCTGGAGTTTGC-3'; IL-6: forward: 5'-ATGCTTCCAATCTGGGTTC-3', reverse: 5'-TGAGGATAATCTTTGCGTTC-3'; TNF- $\alpha$ : forward: 5'-ACGGGCTTTACCTCATCTACTC-3', reverse: 5'-GCTCTTGATGGCAGACAGG-3'. Reverse transcription was performed at $50^{\circ} \mathrm{C}$ for $20 \mathrm{~min}$, and pre-denaturation was performed at $95^{\circ} \mathrm{C}$ for $2 \mathrm{~min}$. The following steps were repeated for 45 cycles: denaturation at $95^{\circ} \mathrm{C}$ for $15 \mathrm{~s}$, annealing at $55^{\circ} \mathrm{C}$ for $25 \mathrm{~s}$, and extension at $72^{\circ} \mathrm{C}$ for $50 \mathrm{~s}$. The expression of each target gene was measured using the $2 \Delta \Delta \mathrm{Cq}$ method [4]. Fold change of each target gene $=$ expression of target mRNA/expression of glyceraldehyde 3-phosphate dehydrogenase (GAPDH) mRNA. GAPDH was used as an internal control. Each experiment was repeated three times independently.

\section{Protein extraction and western blotting analysis}

The cells were cultured and grouped as described previously. Subsequently, the cells were lysed with RIPA buffer (No. R0010, Solarbio), the protein samples in the supernatant were collected after centrifugation at $12,000 \mathrm{rpm}$ for $10 \mathrm{~min}$, and the concentration of protein was detected using a BCA assay (No. PC0020, Solarbio). SDS-PAGE electrophoresis was performed with $60 \mu \mathrm{g}$ of protein, followed which proteins were transferred onto a PVDF membrane. Membranes were then blocked with 5\% skim milk and incubated with the following primary antibodies (1 : 1000) (BDNF (ab108319), GAPDH (ab8245), p-TrkB (ab229908), TrkB (ab134155), p-PKC (ab109539), PKC (ab181558), CaMKII (ab134041), AC-3 (ab125093), RhoA (ab187027), ROCK (ab45171), EGF (ab184265), NT-3 (ab263864) and NT-4 (ab150437)) overnight at $4^{\circ} \mathrm{C}$. Following incubation, the membranes were incubated with secondary antibody at room temperature for $4 \mathrm{~h}$. Protein expression was detected using a chemiluminescent immunoassay (SW2020, Solarbio), and the grey values of proteins were analysed using Image-Pro Plus (version 6.0). Fold change of each target protein = grey value of target protein/grey value of GAPDH.

\section{Enzyme-linked immunosorbent assay}

Enzyme-linked immunosorbent assay (ELISA) was performed according to the protocols of the ELISA kits (mouse IL-1 $\beta$ (ab197742), IL-6 (ab222503), TNF- $\alpha$ (ab208348) and calcium (ab102505) kits). Blood samples were first collected into a $1.5 \mathrm{ml}$ tube, and after clot formation, samples were centrifuged at $2000 \mathrm{~g}$ for $10 \mathrm{~min}$. The culture medium of cells, brain tissue lysates and serum samples of mice were collected and added into each well of a 96-well plate and incubated at room temperature for $1 \mathrm{~h}$. After washing with washing buffer, the samples were incubated with TMB solution for $10 \mathrm{~min}$ at room temperature. Stop solution was added and the OD value was detected at $450 \mathrm{~nm}$ using SpectraMax iD3.

\section{Ethics statement}

Animal studies were performed in accordance with the guidelines of the Declaration of Helsinki and according to the principles and procedures outlined in the National Institutes of Health Guide for the Care and Use of Animals. Animal experiments were performed in the Third Hospital of Heilongjiang Province and were approved by the Health Animal Care and Use Committee of the Third Hospital of Heilongjiang Province (SCXK 2020-0016 of July 2020).

\section{Statistical analysis}

Experimental data are presented as the mean \pm SD, and each experiment was repeated three times independently. One-way ANOVA was performed to detect the differences between groups followed by Tukey's post hoc test [degree of freedom (DF) $n=3$, DF $d=8)$. Significant difference was set at $p<0.05$.

\section{Results \\ Mouse behaviour tests}

As shown in Figure 2A, the percentage of sucrose consumption in the CG, LG, OG and LO groups was $1.00 \pm 0.08,0.62 \pm 0.03,1.03 \pm 0.09$, and $0.94 \pm 0.06$, 
respectively. The percentage of sucrose consumption was significantly lower in the LG group than in the other three groups ( $p=0.0003$, DF $=22.5)$. The immobility time in these groups was $65.2 \pm 3.5,113.6 \pm 5.2$, $58.3 \pm 2.9$, and $74.4 \pm 4.6 \mathrm{~s}$, respectively. The immobility time in the LG group was significantly increased compared to the CG group, and was significantly decreased in the OG and LO groups ( $p=0.0001$, $\mathrm{DF}=106.4$ ) (Fig. 2B). The number of locomotor crossings in these groups was $92.3 \pm 7.3,63.4 \pm 5.2,97.1$ \pm 7.6 , and $86.6 \pm 6.9$, respectively. The number of locomotor crossings was significantly decreased in the LG group compared to the CG group, and was significantly increased in the OG and LO groups compared to the LG group ( $p=0.0014, \mathrm{DF}=14.4)$ (Fig. $2 \mathrm{C}$ ).

\section{Expression of pro-inflammation cytokines in the brain tissues of the mice model of depression}

As shown in Figure 3, the expression of IL-1 $\beta$ in the CG, LG, OG and LO groups of MDD mice was $1.00 \pm 0.08$, $1.32 \pm 0.12,0.94 \pm 0.07$, and $1.06 \pm 0.10$, respectively. The expression of IL-1 $\beta$ was significantly increased in the LG group compared to the CG group, and was significantly decreased in the OG and LO groups compared to the LG group ( $p=0.0053, \mathrm{DF}=9.412$ ) (Fig. 3A). The expression of IL- 6 in each group of MDD mice was $1.00 \pm 0.07,1.26 \pm 0.11,1.03 \pm 0.09$, and 1.01 \pm 0.09 , respectively. The expression of IL- 6 was significantly increased in the LG group compared to the CG group, and was significantly decreased in the OG and LO groups compared to the LG group ( $p=0.0234$, $D F=5.554)$ (Fig. 3B). The expression of TNF- $\alpha$ in each group of MDD mice was $1.00 \pm 0.06,1.42 \pm 0.15,0.91$ \pm 0.05 , and $1.06 \pm 0.06$, respectively. The expression of TNF- $\alpha$ was significantly increased in the LG group compared to the CG group, and was significantly decreased in the OG and LO groups $(p=0.0006$, $\mathrm{DF}=18.64$ ) compared to the LG group (Fig. 3C).

\section{Detection of pro-inflammation cytokines in serum samples of the mice model of depression}

As shown in Figure 4, the concentration of IL-1 $\beta$ in the serum samples of CG, LG, OG, and LO groups of MDD mice was $28.6 \pm 1.8,56.1 \pm 3.5,25.8 \pm 1.7$,
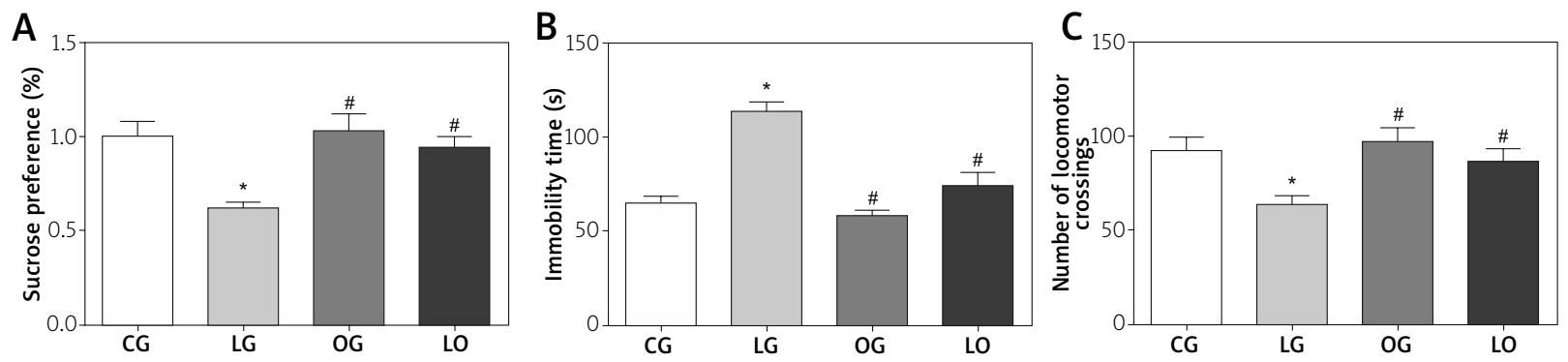

Fig. 2. Results of behaviour tests in the mice model of depression. A) Percentage of sucrose consumption in the sucrose preference test. B) Immobility time in the forced swimming test. C) Number of crossings in the open field test. ${ }^{*} p<0.05$ compared with the CG group, ${ }^{\#} p<0.05$ compared with the LG group. Each experiment was repeated three times independently $(n=10)$, Tukey's post-hoc test was used to detect the difference between groups.
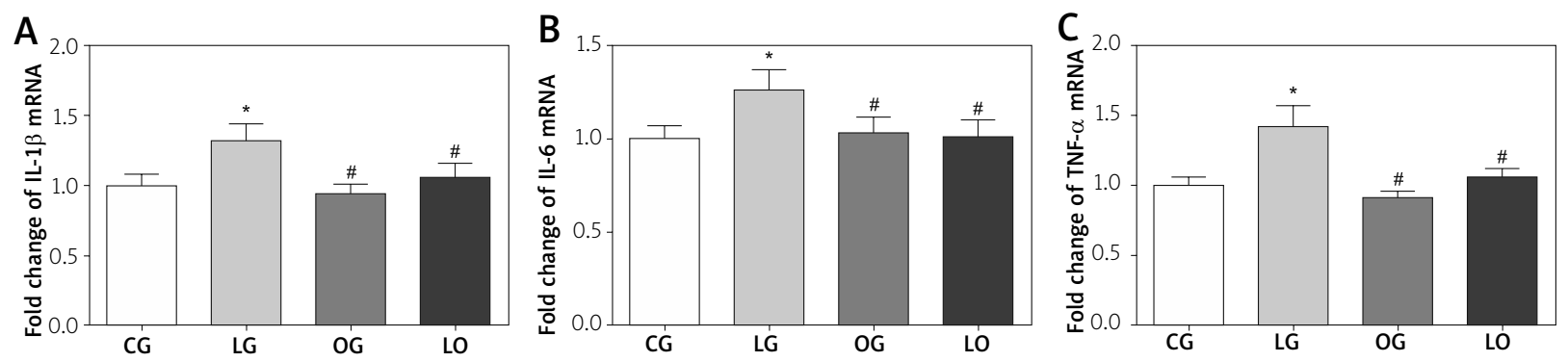

Fig. 3. Expression of IL-1 $\beta, I L-6$ and TNF- $\alpha$ in brain tissue of mice. ${ }^{*} p<0.05$ compared with the CG group, ${ }^{\#} p<0.05$ compared with the LG group. Each experiment was repeated three times independently $(n=10)$, Tukey's post-hoc test was used to detect the difference between groups. 

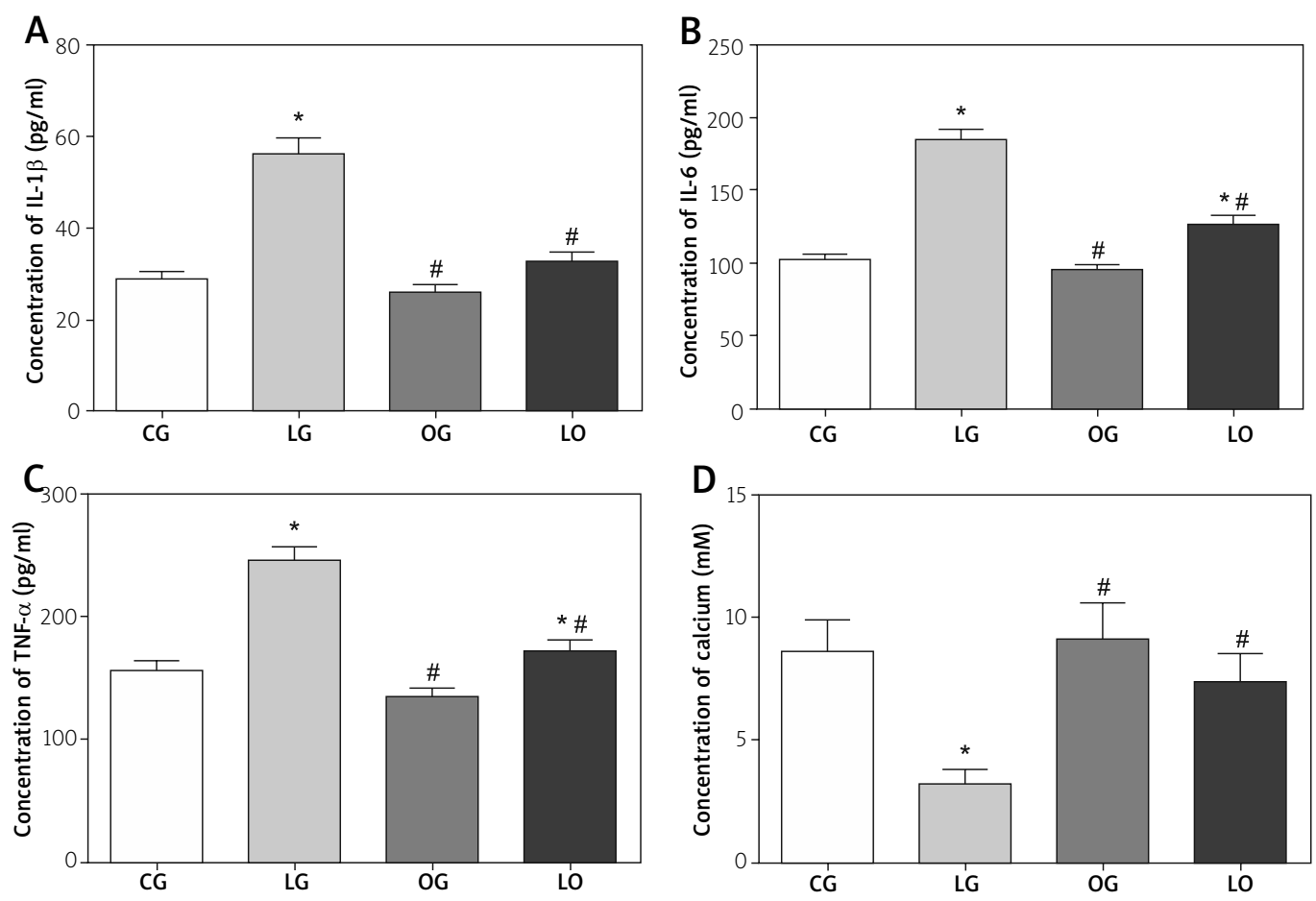

Fig. 4. Concentration of pro-inflammation cytokines and calcium in the mice model of depression. A) Concentration of IL-1 $\beta$ in the serum sample of mice. B) Concentration of IL- 6 in the serum sample of mice. C) Concentration of TNF- $\alpha$ in the serum sample of mice. D) Concentration of intracellular calcium in brain tissue of mice. ${ }^{*} p<0.05$ compared with the CG group, ${ }^{\#} p<0.05$ compared with the LG group. Each experiment was repeated three times independently $(n=10)$, Tukey's post-hoc test was used to detect the difference between groups.

and $32.6 \pm 2.1 \mathrm{pg} / \mathrm{ml}$, respectively. The concentration of IL-1 $\beta$ was significantly increased in the LG group compared to the CG group, and was significantly decreased in the OG and LO groups compared to the LG group ( $p=0.0001, \mathrm{DF}=100.8$ ) (Fig. 4A). The concentration of IL- 6 in these groups was 102.1 $\pm 4.2,184.5 \pm 6.8,95.3 \pm 3.7$, and $126.4 \pm 5.6 \mathrm{pg} / \mathrm{ml}$, respectively. The concentration of IL- 6 was significantly increased in the LG and LO groups and was significantly decreased in the LG and LO groups compared to the CG group ( $p=0.0001, \mathrm{DF}=181.1$ ) (Fig. 4B). The concentration of TNF- $\alpha$ in these groups was 156.3 $\pm 8.2,246.1 \pm 11.3,134.5 \pm 7.4$, and $172.1 \pm 8.9 \mathrm{pg} / \mathrm{ml}$, respectively. The concentration of TNF- $\alpha$ was significantly increased in the $L G$ group compared to the CG group and was significantly decreased in the OG and LO groups compared to the LG group ( $p=0.0001$, DF $=85.54$ ) (Fig. 4C). The concentration of calcium in the brain tissues from these groups was $8.6 \pm 1.3$, $3.2 \pm 0.6,9.1 \pm 1.5$, and $7.4 \pm 1.1 \mathrm{mM}$, respectively. The concentration of calcium was significantly de- creased in the LG group compared to the CG group, and the concentration of calcium was significantly increased in the OG and LO groups compared to the CG group ( $p=0.001$, DF = 15.64) (Fig. 4D). These results showed that the secretion of pro-inflammation cytokines was increased after LPS treatment and decreased after oleuropein stimulation, indicating that oleuropein had anti-inflammation effects. Oleuropein stimulation also increased the concentration of calcium in serum samples, which is another possible mechanism contributing to the improvement of MDD.

\section{Detection of the cellular viability rate and model constructs}

As shown in Figure $5 \mathrm{~A}$, the viability rate of cells in the L, G, O and I groups of the cell model was $100.0 \pm 7.1 \%$, $123.3 \pm 8.0 \%, 147.6 \pm 12.4 \%$, and $103.2 \pm 7.5 \%$, respectively. The viability rate was significantly increased in the $G$ and $O$ groups compared to the $L$ group and was significantly increased in the $\mathrm{O}$ group and significantly decreased in the I group compared to the G group 

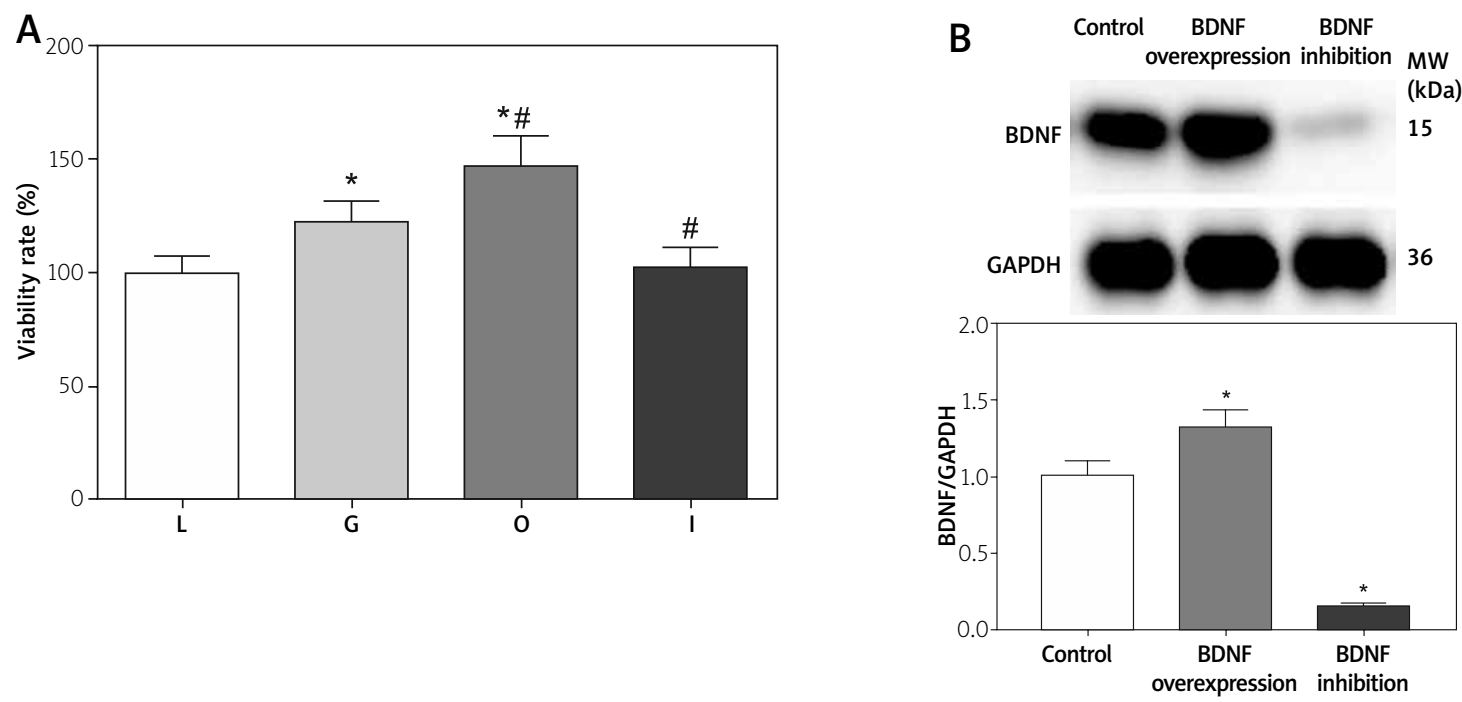

Fig. 5. Expression of BDNF in BDNF overexpression and BDNF inhibition model and detection of cellular viability in each group of the cell model of depression. A) Viability rate of cells in each group of the cell model of depression. * $p<0.05$ compared with the L group, \#p $<0.05$ compared with the G group. B) Expression of BDNF in BDNF overexpression and BDNF inhibition group of cells. ${ }^{*} p<0.05$ compared with the control group. Each experiment was repeated three times independently $(n=3)$, Tukey's post-hoc test was used to detect the difference between groups.

$(p=0.0007, \mathrm{DF}=17.91)($ Fig. $5 \mathrm{~A})$. The expression of BDNF in the control, BDNF overexpression, and BDNF inhibition groups was $1.01 \pm 0.08,1.33 \pm 0.11$, and $0.16 \pm 0.01$, respectively (Fig. $5 \mathrm{~B}$ ). The expression of BDNF was significantly increased in the BDNF overexpression group and was significantly decreased in the BDNF inhibition group $(p=0.0001, \mathrm{DF}=176.9)$.

\section{Expression of pro-inflammation cytokines in each cell model of depression}

As shown in Figure 6, the expression of IL-1 $\beta$ in the L, G, $O$ and I groups was $1.00 \pm 0.07,0.82 \pm 0.06$, $0.51 \pm 0.04$, and $1.06 \pm 0.09$, respectively. The expression of IL-1 $\beta$ was significantly decreased in the $G$ and $O$ groups compared to the L group, and was significantly decreased in the $\mathrm{O}$ group and significantly increased in the I group compared to the $L$ group $(p=0.0001$, $\mathrm{DF}=40.24)($ Fig. $6 \mathrm{~A})$. The expression of IL-6 in these groups was $1.00 \pm 0.08,0.84 \pm 0.06,0.56 \pm 0.04$, and $1.04 \pm 0.08$, respectively. The expression of IL- 6 was significantly decreased in the $\mathrm{O}$ group compared to the L group, was significantly decreased in the $\mathrm{O}$ group and was significantly increased in the I group compared to the $\mathrm{G}$ group ( $p=0.0001$, DF = 31.64) (Fig. 6B). The expression of TNF- $\alpha$ in these groups was $1.00 \pm 0.11$, $0.76 \pm 0.07,0.42 \pm 0.05$ and $0.94 \pm 0.09$, respectively.
The expression of TNF- $\alpha$ was significantly decreased in the $\mathrm{G}$ and $\mathrm{O}$ groups compared to the $\mathrm{L}$ group, and was significantly decreased in the $\mathrm{O}$ group compared to the $\mathrm{G}$ group $(p=0.0001, \mathrm{DF}=29.57)(\mathrm{Fig} .6 \mathrm{C})$.

\section{Concentration of pro-inflammation cytokines in the culture medium of the cell model of depression}

As shown in Figure 7, the concentration of IL-1 $\beta$ in the culture medium of the L, G, O, and I groups of the cell model was $75.1 \pm 10.3,52.3 \pm 8.5,30.4 \pm 5.6$ and $68.6 \pm 9.6 \mathrm{pg} / \mathrm{ml}$, respectively. The concentration of IL-1 $\beta$ was significantly decreased in the $G$ and $O$ groups compared to the $C$ group and was significantly decreased in the $\mathrm{O}$ group compared to the $\mathrm{G}$ group $(p=0.001, \mathrm{DF}=15.78)$ (Fig. 7A). The concentration of IL- 6 in these groups was $365.2 \pm 18.7,308.6 \pm 16.4$, $236.4 \pm 13.6$, and $352.8 \pm 18.0 \mathrm{pg} / \mathrm{ml}$, respectively. The concentration of IL- 6 was significantly decreased in the $\mathrm{G}$ and $\mathrm{O}$ groups compared to the $\mathrm{L}$ group, and was significantly decreased in the $\mathrm{O}$ group and increased in the I group compared to the G group ( $p=0.0001$, $\mathrm{DF}=36.06)$ (Fig. 7B). The concentration of TNF- $\alpha$ in these groups was $532.3 \pm 32.1,463.7 \pm 27.4,382.4$ \pm 21.3 , and $520.6 \pm 31.6 \mathrm{pg} / \mathrm{ml}$, respectively. The concentration of TNF- $\alpha$ was significantly decreased in the $O$ group compared to the $C$ and $G$ groups 

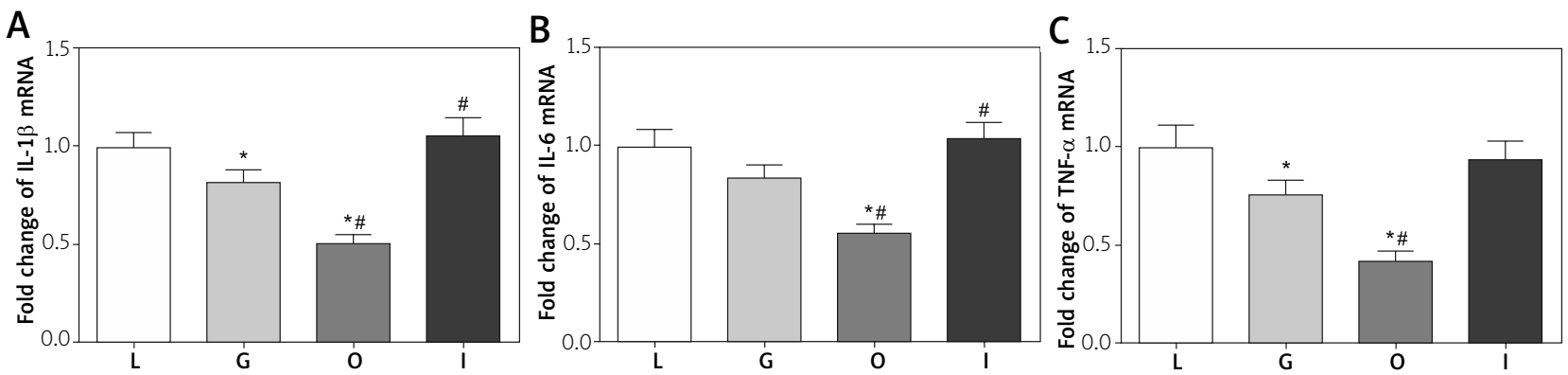

Fig. 6. Expression of IL-1 $\beta$, IL- 6 and TNF- $\alpha$ in each group of the cell model of depression. ${ }^{*} p<0.05$ compared with the $L$ group, ${ }^{\#} p<0.05$ compared with the $G$ group. The expression level of each gene was calculated using $2-{ }^{\Delta \Delta} \mathrm{Cq}$ method. Each experiment was repeated three times independently $(n=3)$, Tukey's post-hoc test was used to detect the difference between groups.
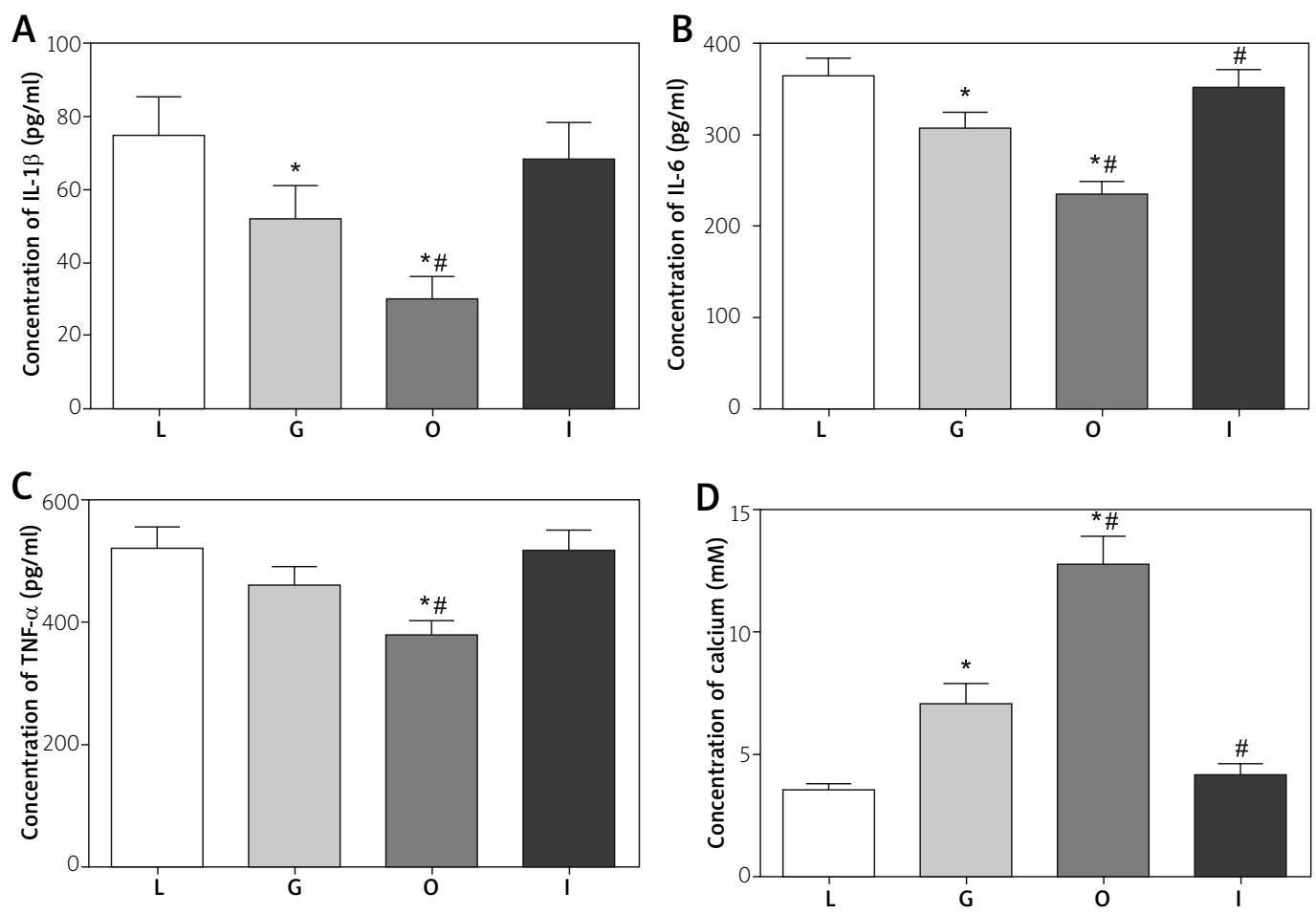

Fig. 7. Concentration of pro-inflammation cytokines and calcium in each group of the cell model of depression. A) Concentration of IL-1 $\beta$ in each group of the cell model of depression. B) Concentration of IL- 6 in each group of the cell model of depression. C) Concentration of TNF- $\alpha$ in each group of the cell model of depression. D) Concentration of intracellular calcium in each group of the cell model of depression. ${ }^{*} p<0.05$ compared with the L group, ${ }^{\#} p<0.05$ compared with the $\mathrm{G}$ group. Each experiment was repeated three times independently $(n=3)$, Tukey's post-hoc test was used to detect the difference between groups.

$(p=0.0007, \mathrm{DF}=17.4)$ (Fig. 7C). The concentration of calcium in these groups of cell lysates was $3.6 \pm 0.2$, $7.1 \pm 0.8,12.8 \pm 1.1$, and $4.2 \pm 0.4 \mathrm{mM}$, respectively. The concentration of calcium was significantly increased in the $\mathrm{G}$ and $\mathrm{O}$ groups compared to the $\mathrm{L}$ group, and was significantly increased in the $\mathrm{O}$ group and significantly decreased in the I group compared to the $\mathrm{G}$ group $(p=0.0001, \mathrm{DF}=103.5)($ Fig. 7D).

\section{Expression of the BDNF/TrkB/CaMKII signalling pathway in each cell model of depression}

As shown in Figure 8 , the expression of BDNF in the L, G, $O$ and I groups of the cell model was $0.48 \pm 0.04$, $1.01 \pm 0.08,1.80 \pm 0.15$, and $0.83 \pm 0.07$, respectively. The expression of BDNF was significantly increased in 

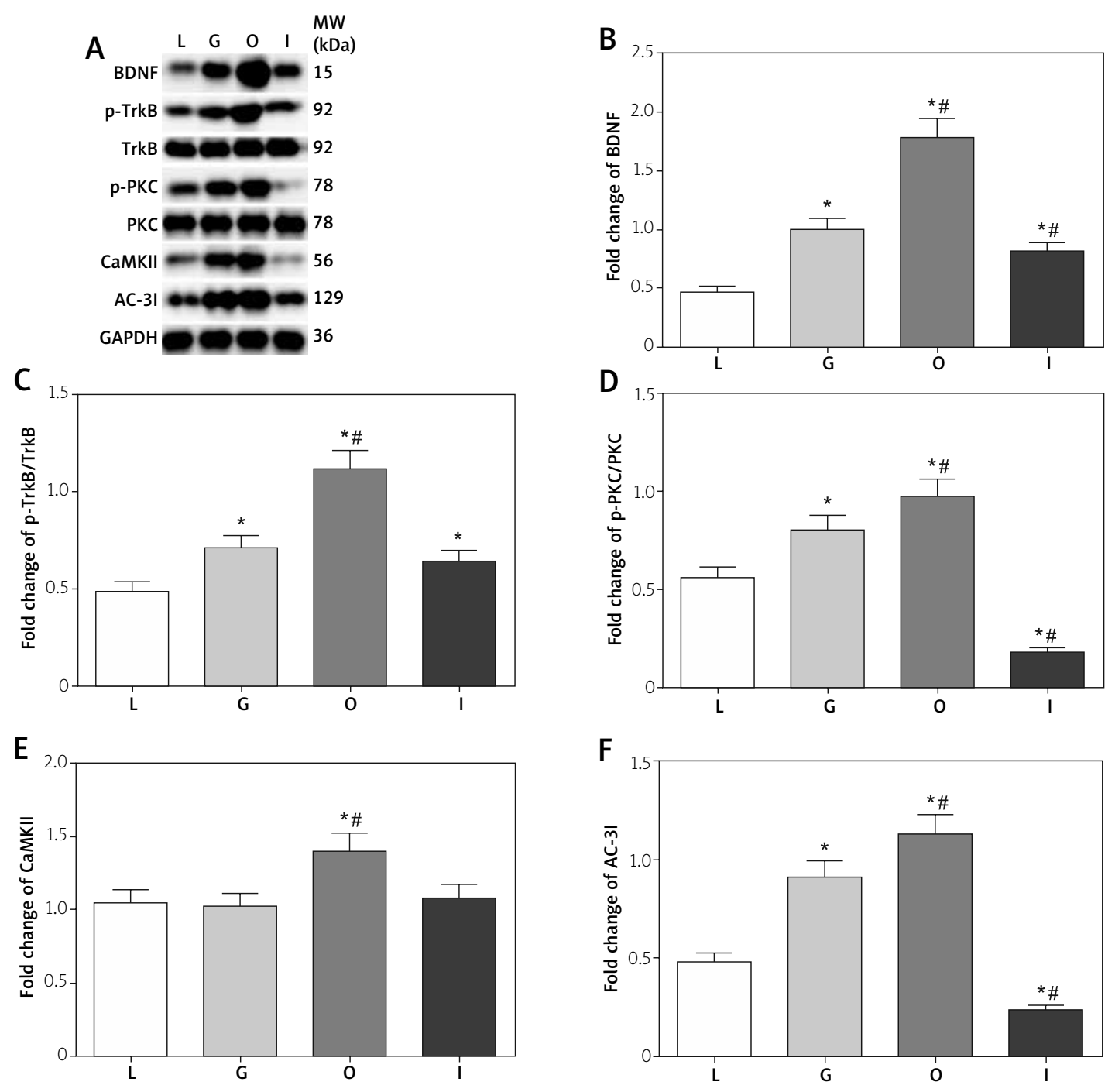

Fig. 8. Expression of key members of the calcium regulation pathway in the cell model of depression. A) Detection of BDNF, TrkB, p-PKC, PKC, CaMKII and AC-3I in each group of the cell model of depression. B) Quantitative analysis of each target protein. ${ }^{*} p<0.05$ compared with the $\mathrm{L}$ group, ${ }^{*} p<0.05$ compared with the $\mathrm{G}$ group. Each experiment was repeated three times independently $(n=3)$, Tukey's post-hoc test was used to detect the difference between groups.

all treatment groups compared to the L group, and the expression of BDNF was significantly increased in the $\mathrm{O}$ group and significantly decreased in the I group compared to the $\mathrm{G}$ group ( $p=0.0001, \mathrm{DF}=105.7)$ (Fig. 8B). The ratio of $\mathrm{p}-\mathrm{TrkB} / \mathrm{TrkB}$ in these groups was $0.49 \pm 0.04$, $0.72 \pm 0.06,1.12 \pm 0.09$, and $0.64 \pm 0.05$, respectively, and the ratio of $\mathrm{p}$-TrkB/TrkB was significantly increased in all treatment groups compared to the L group, and was significantly increased in the $\mathrm{O}$ group compared to the $G$ group ( $p=0.0001, D F=55.01)$ (Fig. 8C). The ratio of $p$-PKC/PKC in these groups was $0.57 \pm 0.05$, $0.81 \pm 0.07,0.98 \pm 0.08$, and $0.19 \pm 0.02$, respectively.
The ratio of p-PKC/PKC was significantly increased in the $G$ and $O$ groups, and significantly decreased in the I group compared to the L group, and was significantly increased in the $\mathrm{O}$ group and significantly decreased in the I group compared to the G group $(p=0.0001$, $\mathrm{DF}=99.12)($ Fig. 8D). The expression of CaMKII in these groups was $1.05 \pm 0.09,1.03 \pm 0.09,1.41 \pm 0.12$, and $1.08 \pm 0.09$, respectively. The expression of CaMKII was significantly increased in the $\mathrm{O}$ group compared to the $L$ and $G$ group ( $p=0.0044, D F=9.992$ ) (Fig. 8E). The expression of AC3 in these groups was $0.48 \pm 0.04$, $0.92 \pm 0.08,1.14 \pm 0.09$, and $0.24 \pm 0.02$, respectively. 

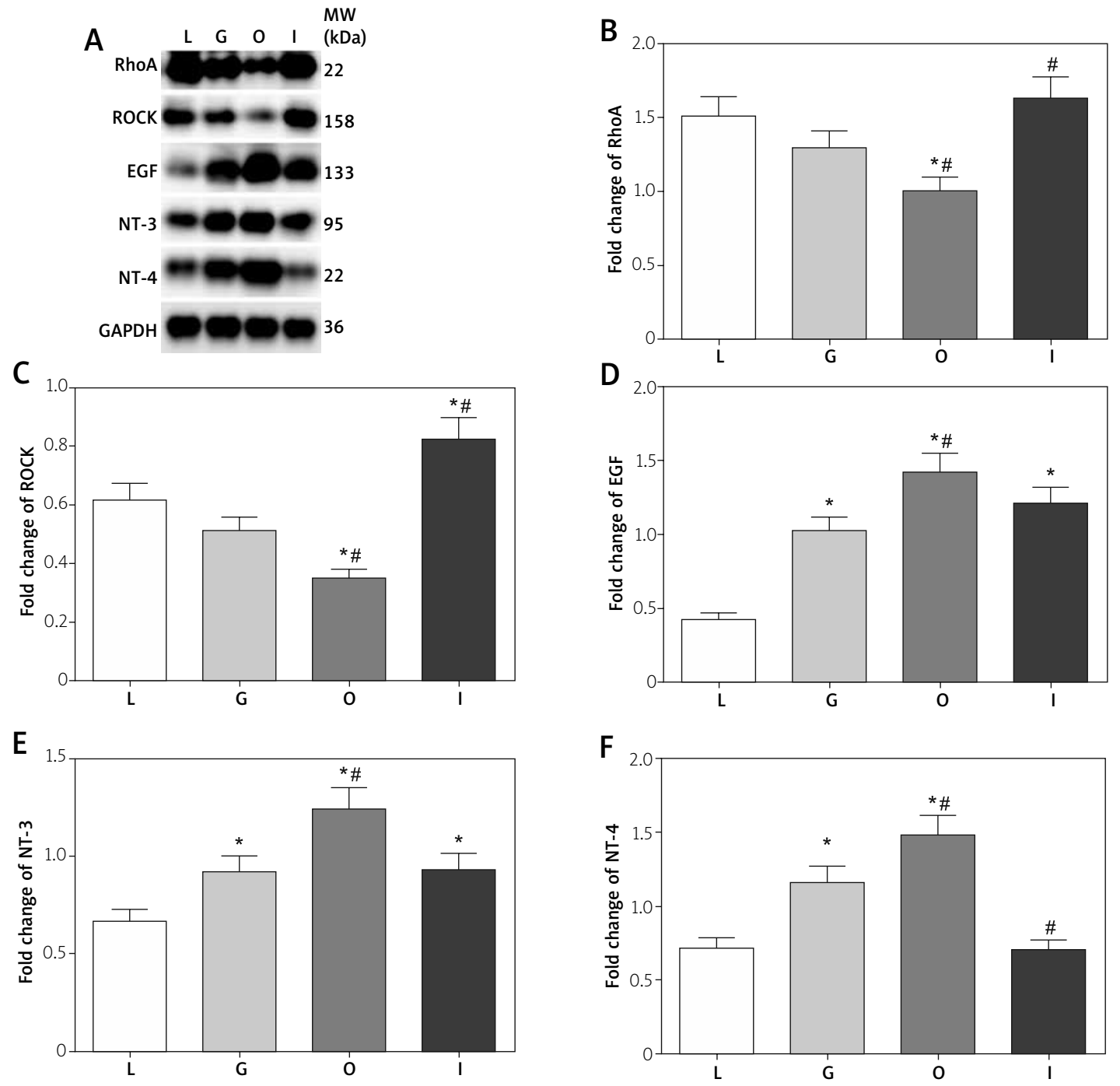

Fig. 9. Expression of key members of the RhoA/ROCK signalling pathway in the cell model of depression. A) Expression of RhoA, ROCK, EGF, NT-3 and NT-4 in each group of the cell model of depression. B) Quantitative analysis of each target protein. ${ }^{*} p<0.05$ compared with the $\mathrm{L}$ group, ${ }^{*} p<0.05$ compared with the $\mathrm{G}$ group. Each experiment was repeated three times independently $(n=3)$, Tukey's post-hoc test was used to detect the difference between groups.

The expression of AC3 was significantly increased in the $\mathrm{G}$ and $\mathrm{O}$ group compared to L group, and significantly increased in the $O$ group and decreased in the I group ( $p=0.0001, \mathrm{DF}=121.7$ ) compared to the G group (Fig. 8F).

\section{Expression of the RhoA/ROCK signalling pathway in each cell model of depression}

As shown in Figure 9, the expression of RhoA in the L, G, O and I groups of the cell model was $1.52 \pm 0.13,1.31 \pm 0.11,1.01 \pm 0.08$, and $1.64 \pm 0.14$, respectively. The expression of RhoA was significantly decreased in the $O$ group compared to the $L$ group, and significantly decreased in the $O$ group and significantly increased in the I group compared to the $\mathrm{G}$ group ( $p=0.0008, \mathrm{DF}=16.63$ ) (Fig. 9B). The expression of ROCK in these groups was $0.62 \pm 0.05$, $0.52 \pm 0.04,0.35 \pm 0.03$, and $0.83 \pm 0.07$, respectively. The expression of ROCK was significantly decreased in the $O$ group and significantly increased in the I group $(p=0.0001, \mathrm{DF}=48.73)$ compared to the $L$ and $G$ groups (Fig. 9C). The expression of epidermal 
growth factor (EGF) in these groups was $0.43 \pm 0.04$, $1.03 \pm 0.09,1.43 \pm 0.12$, and $1.22 \pm 0.10$, respectively. The expression of EGF was significantly increased in all treatment groups compared to the L group, and the expression of EGF was significantly increased in the $\mathrm{O}$ group compared to the $\mathrm{G}$ group $(p=0.0001$, $\mathrm{DF}=65.23$ ) (Fig. 9D). The expression of NT-3 in these groups was $0.67 \pm 0.06,0.92 \pm 0.08,1.25 \pm 0.10$, and $0.94 \pm 0.08$, respectively. The expression of NT-3 was significantly increased in all treatment groups compared to the L group, and the expression of NT-3 was significantly increased in the $\mathrm{O}$ group compared to the $\mathrm{G}$ group $(p=0.0002, \mathrm{DF}=25.65)$ (Fig. 9E). The expression of NT-4 in these groups was $0.72 \pm 0.06$, $1.17 \pm 0.10,1.49 \pm 0.12$, and $0.71 \pm 0.06$, respectively. The expression of NT-4 was significantly increased in the $\mathrm{G}$ and $\mathrm{O}$ groups compared to the $\mathrm{L}$ group, and was significantly increased in the $\mathrm{O}$ group and significantly decreased in the I group compared to the G group ( $p=0.0001, \mathrm{DF}=54.36$ ) (Fig. 9F).

\section{Discussion}

Brain-derived neurotrophic factor is a secreted protein belonging to the neurotrophin family. BDNF was first purified from the brain tissue of pigs and has been found to support the survival and differentiation of neurons [4]. In the central nervous system, BDNF protects neuronal cells from apoptosis, and increases the synaptic function and morphology of neuronal cells [34]. Loss of BDNF expression induces spinal atrophy via impairment of the GABAergic system [37]. We first found that overexpression of BDNF promotes cell proliferation under LPS stimulation using an in vitro model. Moreover as the inflammatory response process is activated in the MDD model, the expression of pro-inflammatory cytokines including IL-1 $\beta$, IL-6, and TNF- $\alpha$, was significantly increased after LPS stimulation, and was significantly reduced after BDNF overexpression combined with oleuropein, this effect was also detected in a mouse model. Inflammation is critical for the development of psychiatric disorders in nervous system diseases, because the inflammation process affects the peripheral immune system [14] The increased expression of pro-inflammatory cytokines in the CNS leads to the activation of microglia and astrocytes and further continuous production of these cytokines [40], resulting in the MDD and Alzheimer's disease. In patients with MDD, the concentrations of IL-1 $\beta, \mathrm{IL}-6$, and TNF- $\alpha$ are elevated, which can lead to late-life depression [33], thus, a reduction in the concentration of IL-1 $\beta, I L-6$, and TNF- $\alpha$ may have a therapeutic effect in MDD. Here, the combination of BDNF and oleuropein treatment significantly reduced the expression and secretion of IL- $1 \beta$, IL- 6 , and TNF- $\alpha$, suggesting that this treatment might be effective in the context of MDD.

BDNF is a major neurotrophic factor that regulates the proliferation and differentiation of neuronal cells. The biological function of BDNF depends on binding to TrkB, and, according to a previous study, administration of LPS or pro-inflammation cytokines led to a significant reduction in BDNF expression [48]. Moreover, a previous study suggested that these effects might be mediated by the BDNF/TrkB signalling pathway, and this interaction has been proven to promote synaptic efficiency and long-term potentiation [47]. Indeed, neuronal cell function is promoted when TrkB is overexpressed [32]. In addition, a previous study also found that the release of neurotransmitters is regulated by PKC, a downstream molecule of the TrkB signalling pathway. BDNF/TrkB regulates the activity of neuronal cells in an intracellular calcium-dependent manner at the release site of BDNF [6]. Here, we found that after oleuropein treatment, the expression of BDNF and TrkB was increased, and was further increased after overexpression of BDNF. BDNF/TrkB induces sustained calcium influx through canonical transient receptor potential channels, mediated by TrkB-PKC activation, which promotes the release of calcium from internal calcium stores through activation of CaMKII and other calcium-dependent signalling steps [27]. CaMKII has been reported to regulate the oxidative stress and anti-depressant response, and, a recent study reported that CaMKII is also associated with the development of MDD [28]. Thus, we considered that increased expression of BDNF/TrkB might induce the expression of CaMKII, which leads to the increased concentration of calcium in serum samples of MDD mice, and that this effect might contribute to alleviation of MDD symptoms. Adenylyl cyclase 3 (AC3) is considered a critical enzyme in the regulation of the CAMP signalling pathway in neuronal cells [15]. Membrane-associated ACs are divided into four groups, and AC3 belongs to class I, which is a calcium/calmodulin-sensitive form [44]. Using a genome-wide association method, researchers found that $A C 3$ is one of the top-ranked genes 
for the development of MDD [45], and strong depression-like behaviour was found to be present in $A C 3$ knockout mice [43]. Oleuropein treatment led to an increase in $A C 3$ expression and the expression was enhanced after overexpression of BDNF, indicating that the combination of oleuropein treatment and BDNF overexpression might contribute to the therapeutic effect of MDD, potentially via activation of the BDNF/TrkB/PKC signalling pathway.

Ras homolog gene family, member A (RhoA) is a member of the small GTPase protein family. Rho-associated protein kinase (ROCK) is a downstream effector of RhoA. RhoA/ROCK plays a critical role in cellular growth, transformation and cytoskeleton regulation [5]. Activation of the RhoA/ROCK signalling pathway is commonly observed in the regulation of CNS injury. A previous study found that the activation of RhoA started after $24 \mathrm{~h}$, reached a peak after 3 days of injury and decreased until day 7 in a model of TBI [11]. A similar result was observed in a model of spinal cord injury (SCl), and researchers found that high expression of the RhoA/ROCK signalling pathway persisted for 3 months [12]. In addition, a recent study observed activation of the RhoA/ ROCK signalling pathway in a seizure model induced by kainic acid, which is associated with axonal and synaptic retraction [11]. Thus, we hypothesized that the RhoA/ROCK signalling pathway plays an important role in the regulation of MDD.

Epidermal growth factor promotes the survival and proliferation of neural cells and activates growth factor signal transduction by binding to receptors. EGF knockout mice present behavioural abnormalities, including increased locomotor activity, damage to prepulse inhibition, and deficits in short-term memory. In addition, EGF knockout also reduces the activation of CaMKII and p21-activated kinase [35]. However, only a few studies focused on the function of EGF in patients with MDD, a previous study found that the concentration of EGF in plasma was significantly lower in patients with MDD [41], while we found that the expression of EGF was significantly increased after treatment with oleuropein combined with BDNF overexpression, which may contribute to reducing the inflammatory response after LPS stimulation. Thus, it is conceivable that a reduction in the inflammatory response might lead to a reduction in the symptoms of MDD. Neurotrophins (NTs) are one of the most studied factors in the brain, among which, NT-3 and NT-4 are most commonly observed
[20]. NT-3 plays an important role in the development of excitatory synapses, and it has been previously shown that presynaptic differentiation of NT-3 occurs in a dose-independent manner [16]. In addition, using an in vitro model, researchers found that NT-3 promotes the survival of sympathetic neuroblasts, which further develop into sympathetic precursors [9]. Moreover, another NT member NT-4 can induce the differentiation of multiple neuronal cells, including cholinergic neurons, hippocampal neurons, and cerebellar granule cells [19], as well as improve the survival of trigeminal and jugular neurons during embryonic development [3]. The RhoA/ROCK signalling pathway has been shown to regulate the growth and differentiation of neuronal cells by regulating the expression of NGF, NT-3, and NT-4. Knockout of RhoA has been shown to enhance the outgrowth lengths of neurites compared to the control group, and inhibition of ROCK also promoted axon/neurite outgrowth by blocking the activation of the growth inhibitory pathway [18]. Here, we found that the expression of both NT-3 and NT-4 increased after treatment with oleuropein combined with BDNF overexpression, which may directly contribute to the protective effect in neuronal cells. Moreover, we also found that downregulation of RhoA/ROCK expression after treatment with oleuropein combined with BDNF overexpression increased the expression of NT-3 and NT-4, which further reduces the inflammatory response after LPS stimulation in a MDD model. Furthermore, oleuropein combined with BDNF overexpression enhanced the expression of neuron-protective factors via inhibition of the RhoA/ROCK signalling pathway in vivo, which might contribute to the therapeutic effect in MDD mice, and even contribute to the recovery of brain function.

With regards to the limitations of this study, this research was only performed in a mouse model, and the sample size was limited. Therefore, further experiments should be performed using more methods, including Co-IP and ChIP to explore the detailed underlying mechanisms. Moreover, further experiments on the effect of BDNF should be performed using primary neuron cells, as well as in clinical treatment. We intend to perform these experiments in the future to confirm the effect of BDNF in the treatment of MDD, which contributes to the clinical application of BDNF. However, according to a previous study, exogenous BDNF does not directly traverse the blood-brain barrier, which limits the 
therapeutic function of BDNF. In order to solve this problem, some researchers designed a liposome that can carry exogenous BDNF through the bloodbrain barrier, this method will be verified in our further experiments.

\section{Disclosure}

\section{The authors report no conflict of interest.}

\section{References}

1. Abuelezz SA, Hendawy N, Magdy Y. Targeting oxidative stress, cytokines and serotonin interactions via indoleamine 2, 3 dioxygenase by coenzyme Q10: role in suppressing depressive like behavior in rats. J Neuroimmune Pharmacol 2017; 12: 277-291.

2. Abel EL. Behavior and corticosteroid response of Maudsley reactive and nonreactive rats in the open field and forced swimming test. Physiol Behav 1991; 50: 151-153.

3. Altar CA, Boylan CB, Fritsche M, Jackson C, Hyman C, Lindsay RM. The neurotrophins NT-4/5 and BDNF augment serotonin, dopamine, and GABAergic systems during behaviorally effective infusions to the substantia nigra. Exp Neurol 1994; 130: 31-40.

4. Barde YA, Edgar D, Thoenen H. Purification of a new neurotrophic factor from mammalian brain. EMBO J 1982; 1: 549-553.

5. Beher D, Clarke EE, Wrigley JDJ, Martin ACL, Nadin A, Churcher I, Shearman MS. Selected non-steroidal anti-inflammatory drugs and their derivatives target gamma-secretase at a novel site. Evidence for an allosteric mechanism. J Biol Chem 2004; 279: 43419-43426.

6. Brigadski T, Hartmann M, Lessmann V. Differential vesicular targeting and time course of synaptic secretion of the mammalian neurotrophins. J Neurosci 2005; 25: 7601-7614.

7. Bouallagui Z, Han J, Isoda H, Sayadi S. Hydroxytyrosol rich extract from olive leaves modulates cell cycle progression in MCF-7 human breast cancer cells. Food Chem Toxicol 2011; 49 179-184.

8. Burak IA, Bora T, Emre T, Kemal UT, Melis O, Nevin E, Alper B, Kursad G, Sermin G. Melatonin attenuates LPS-induced acute depressive-like behaviors and microglial NLRP3 inflammasome activation through the SIRT1/Nrf2 pathway. Front Immunol 2019; 10: 1511

9. DiCicco-Bloom E, Friedman WJ, Black IB. NT-3 stimulates sympathetic neuroblast proliferation by promoting precursor survival. Neuron 1993; 11: 1101-1111.

10. Duman RS, Li N. A neurotrophic hypothesis of depression: role of synaptogenesis in the actions of NMDA receptor antagonists. Philos Trans R Soc Lond B Biol Sci 2012; 367: 2475-2484.

11. Dubreuil Cl, Marklund N, Deschamps K, McIntosh TK, McKerracher L. Activation of Rho after traumatic brain injury and seizure in rats. Exp Neurol 2006; 198: 361-369.

12. Erschbamer MK, Hofstetter CP, Olson L. RhoA, RhoB, RhoC, Rac1, Cdc42, and Tc10 mRNA levels in spinal cord, sensory ganglia, and corticospinal tract neurons and long-lasting specific changes following spinal cord injury. J Comp Neurol 2005; 484: 224-233.

13. GBD 2016 Disease and Injury Incidence and Prevalence Collaborators. Global, regional, and national incidence, prevalence, and years lived with disability for 328 diseases and injuries for 195 countries, 1990-2016: a systematic analysis for the Global Burden of Disease Study 2016. Lancet 2017; 390: 1211-1259.

14. Gruver AL, Hudson LL Sempowski GD. Immunosenescence of ageing. J Pathol 2007; 211: 144-156.

15. Guadiana SM, Semple-Rowland S, Daroszewski D, Madorsky I, Breunig JJ, Mykytyn K, Sarkisian MR. Arborization of dendrites by developing neocortical neurons is dependent on primary cilia and type 3 adenylyl cyclase. J Neurosci 2013; 33: 2626-2638.

16. Han KA, Woo D, Kim S, Choii G, Jeon S, Won SY, et al. Neurotrophin-3 regulates synapse development by modulating TrkCPTPo synaptic adhesion and intracellular signaling pathways. J Neurosci 2016; 36: 4816-4831.

17. Howren MB, Lamkin DM, Suls J. Associations of depression with C-reactive protein, IL-1, and IL-6: a meta-analysis. Psychosom Med 2009; 71: 171-186.

18. Hu J, Selzer ME. RhoA as a target to promote neuronal survival and axon regeneration. Neural Regen Res 2017; 12: 525-528.

19. Ip NY, Li Y, Yancopoulos GD, Lindsay RM. Cultured hippocampal neurons show responses to BDNF, NT-3, and NT-4, but not NGF. J Neurosci 1993; 13: 3394-3405.

20. Keefe KM, Sheikh IS, Smith GM. Targeting neurotrophins to specific populations of neurons: NGF, BDNF, and NT-3 and their relevance for treatment of spinal cord injury. Int I Mol Sci 2017; 18: E548.

21. Kim MH, Min JS, Lee JY, Chae U, Yang EJ, Song KS, Lee HS, Lee HJ, Lee SR, Lee DS. Oleuropein isolated from fraxinus rhynchophylla inhibits glutamate-induced neuronal cell death by attenuating mitochondrial dysfunction. Nutr Neurosci 2018; 21: 520-528.

22. Kohler O, Krogh J, Mors O, Benros ME. Inflammation in depression and the potential for anti-inflammatory treatment. Curr Neuropharmacol 2016; 14: 732-742.

23. Krisztina M, Mark PM. BDNF mediates adaptive brain and body responses to energetic challenges. Trends Endocrinol Metab 2014; 25: 89-98.

24. Liu Y, Ho RC, Mak A. Interleukin (IL)-6, tumour necrosis factor alpha (TNF-alpha) and soluble interleukin-2 receptors (sIL2R) are elevated in patients with major depressive disorder: A metaanalysis and meta-regression. J Affect Disord 2012; 139: 230-239.

25. Lindholm JSO, Castrén E. Mice with altered BDNF signaling as models for mood disorders and antidepressant effects. Front Behav Neurosci 2014; 8: 143.

26. Livak KJ, Schmittgen TD. Analysis of relative gene expression data using real-time quantitative PCR and the 2(-Delta Delta $C(T)$ ) method. Methods 2001; 25: 402-408.

27. Li W, Calfa G, Larimore J, Pozzo-Miller L. Activity-dependent BDNF release and TRPC signaling is impaired in hippocampal neurons of Mecp2 mutant mice. Proc Natl Acad Sci U S A 2012; 109: 17087-17092.

28. Li K, Zhou T, Liao LJ, Yang ZF, Wong C, Henn F, Malinow R, John RY, $\mathrm{Hu} \mathrm{HL} . \beta \mathrm{CaMKII}$ in lateral habenula mediates core symptoms of depression. Science 2013; 341: 1016-1020. 
29. Milan I, Una JV, Nevena MS, Zoran M, Djurdjica J, Jelica GM, Danijela K, Dragana D. Highly potent antioxidant Olea europaea L. leaf extract affects carotid and renal haemodynamics in experimental hypertension: The role of oleuropein. EXCLI J 2018; 17: 29-44.

30. Michela C, Rosanna DP, Daniela I, Rosalia C, Valeria MM, Antonio P, Enzo P, Domenico B, Angelo P, Emanuela E, Salvatore C. Effects of a polyphenol present in olive oil, oleuropein aglycone, in a murine model of intestinal ischemia/reperfusion injury. J Leukoc Biol 2013; 93: 277-287.

31. Miles EA, Zoubouli P, Calder PC. Differential anti-inflammatory effects of phenolic compounds from extra virgin olive oil identified in human whole blood cultures. Nutrition 2005; 21: 389-394.

32. Michaelsen K, Zagrebelsky M, Berndt-Huch J, Polack M, Buschler A, Sendtner $M$, Korte $M$. Neurotrophin receptors TrkB. T1 and p75NTR cooperate in modulating both functional and structural plasticity in mature hippocampal neurons. Eur J Neurosci 2010; 32: 1854-1865.

33. Mónica MC, Lucía C, John TOB, María-José DG, Cristina BA, Kenji S, Marta A, Ana GP. Peripheral inflammatory parameters in late-life depression: A systematic review. International journal of molecular sciences. Int J Mol Sci 2016; 17: 2022.

34. Nikoletopoulou V, Lickert H, Frade JM, Rencurel C, Giallonardo P, Zhang LX, Bibel M, Barde YA. Neurotrophin receptors TrkA and TrkC cause neuronal death whereas TrkB does not. Nature 2010; 467: 59-63.

35. Oyagi A, Oida Y, Kakefuda K, Shimazawa M, Shioda N, Moriguchi S, Kitaichi K, Nanba D, Yamaguchi K, Furuta Y, Fukunaga K, Higashiyama S, Hara H. Generation and characterization of conditional heparin-binding EGF-like growth factor knockout mice. PLoS One 2009; 4: e7461.

36. Rahimi N, Delfan B, Motamed-Gorji N, Dehpour AR. Effects of oleuropein on pentylenetetrazol-induced seizures in mice: involvement of opioidergic and nitrergic systems. J Nat Med 2017; 71: 389-396.

37. Rauskolb S, Zagrebelsky M, Dreznjak A, Deogracias R, Matsumoto T, Wiese S, Erne B, Sendtner M, Schaeren WN, Korte M, Barde YA. Global deprivation of brain-derived neurotrophic factor in the CNS reveals an area-specific requirement for dendritic growth. J Neurosci 2010; 30: 1739-1749.

38. Sanjana NE, Shalem O, Zhang F. Improved vectors and genomewide libraries for CRISPRscreening. Nat Methods 2014; 11: $783-$ 784.

39. Song C, Manku MS, Horrobin DF. Long-chain polyunsaturated fatty acids modulate interleukin-1beta-induced changes in behavior, monoaminergic neurotransmitters, and brain inflammation in rats. J Nutr 2008; 138: 954-963.

40. Sparkman NL, Johnson RW. Neuroinflammation associated with aging sensitizes the brain to the effects of infection or stress. Neuroimmunomodulation 2008; 15: 323-330.

41. Tian W, Zhang J, Zhang K, Yang H, Sun Y, Shen Y, Xu Q. A study of the functional significance of epidermal growth factor in major depressive disorder. Psychiatr Genet 2012; 22: 161-167.

42. Valkanova V, Ebmeier KP, Allan CL. CRP, IL-6 and depression: A systematic review and metaanalysis of longitudinal studies. J Affect Disord 2013; 150: 736-744.
43. Visel A, Alvarez-Bolado G, Thaller C, Eichele G. Comprehensive analysis of the expression patterns of the adenylate cyclase gene family in the developing and adult mouse brain. J Comp Neurol 2006; 496: 684-697.

44. Wei J, Zhao AZ, Chan GC, Baker LP, Impey S, Beavo JA, Storm DR. Phosphorylation and inhibition of olfactory adenylyl cyclase by CaM kinase II in Neurons: a mechanism for attenuation of olfactory signals. Neuron 1998; 21: 495-504

45. Wray NR, Pergadia ML, Blackwood DHR, Penninx BWJH, Gordon SD, Nyholt DR, Ripke S, Maclntyre DJ, McGhee KA, Maclean AW, Smit JH, Hottenga JJ, Willemsen G, Middeldorp CM, de Geus EJC, Lewis CM, McGuffin P, Hickie IB, van den Oord EJCG, Liu JZ, Macgregor S, McEvoy BP, Byrne BM, Medland SE, Statham DJ, Henders AK, Heath AC, Montgomery GW, Martin NG, Boomsma DI, Madden PAF, Sullivan PF. Genome-wide association study of major depressive disorder: new results, meta-analysis, and lessons learned. Mol Psychiatry 2012; 17: 36-48.

46. Willner P, Towell A, Sampson D, Sophokleous S, Muscat R. Reduction of sucrose preference by chronic unpredictable mild stress, and its restoration by a tricyclic antidepressant. Psychopharmacology (Berl) 1987; 93: 358-364.

47. Zakharenko SS, Patterson SL, Dragatsis I, Zeitlin SO, Siegelbaum SA, Kandel ER, Morozov A. Presynaptic BDNF required for a presynaptic but not postsynaptic component of LTP at hippocampal CA1-CA3 synapses. Neuron 2003; 39: 975-990.

48. Zhang JC, Wu J, Fujita Y, Yao W, Ren Q, Yang C, Li SX, Shirayama Y, Hashimoto K. Antidepressant effects of TrkB ligands on depression-like behavior and dendritic changes in mice after inflammation. Int J Neuropsychopharmacol 2015; 18: pyu077. 